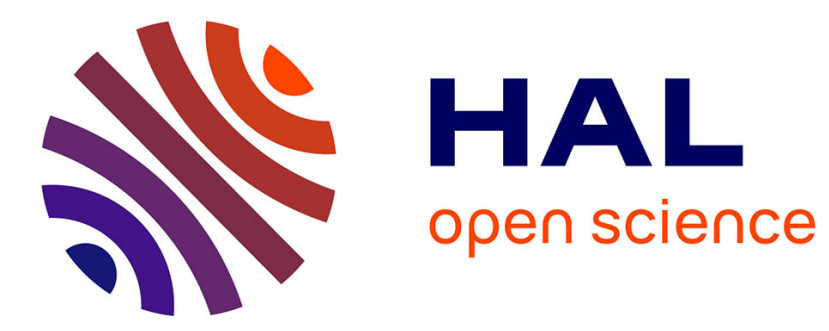

\title{
Concurrent Airframe-Controller Optimization of a Guided Projectile fitted with Lifting Surfaces
}

\author{
Valentin Riss, Emmanuel Roussel, Edouard Laroche
}

\section{To cite this version:}

Valentin Riss, Emmanuel Roussel, Edouard Laroche. Concurrent Airframe-Controller Optimization of a Guided Projectile fitted with Lifting Surfaces. 2022 AIAA SciTech Forum, 3-7 janv. 2022, San Diego, CA, USA, Jan 2022, San Diego, United States. hal-03398233

\section{HAL Id: hal-03398233 https://hal.science/hal-03398233}

Submitted on 22 Oct 2021

HAL is a multi-disciplinary open access archive for the deposit and dissemination of scientific research documents, whether they are published or not. The documents may come from teaching and research institutions in France or abroad, or from public or private research centers.
L'archive ouverte pluridisciplinaire HAL, est destinée au dépôt et à la diffusion de documents scientifiques de niveau recherche, publiés ou non, émanant des établissements d'enseignement et de recherche français ou étrangers, des laboratoires publics ou privés. 


\title{
Concurrent Airframe-Controller Optimization of a Guided Projectile fitted with Lifting Surfaces
}

\author{
Valentin Riss* and Emmanuel Roussel ${ }^{\dagger}$ \\ French-German research institute of Saint-Louis, 68300, Saint-Louis, France \\ Edouard Laroche \\ Laboratoire des sciences de l'ingénieur, de l'informatique et de l'imagerie, 67412, Illkirch, France
}

Innovative long-range guided projectiles concepts require precise flight path tracking in order to achieve maximum range and terminal accuracy. For such airframes featuring large lifting surfaces, vertical maneuvering performance relies on pitch attitude control.

Thus, a challenging problem arises from the conflict between performance requirements and actuator limitations. The projectile static stability should be tailored to limit controls deflections and actuator bandwidth. This work proposes a plant-controller optimization (PCO) procedure to tune the projectile static stability and the controller gains in a single step. This methodology is applied to actuator usage minimization under control performance constraint. The optimal airframe-controller design shows superior actuator roll-off characteristics compared to the outcome of the traditional "design then control" methodology. The codesign also proves to be much less computationally intensive, which confirms the relevance of the approach.

\section{Nomenclature}

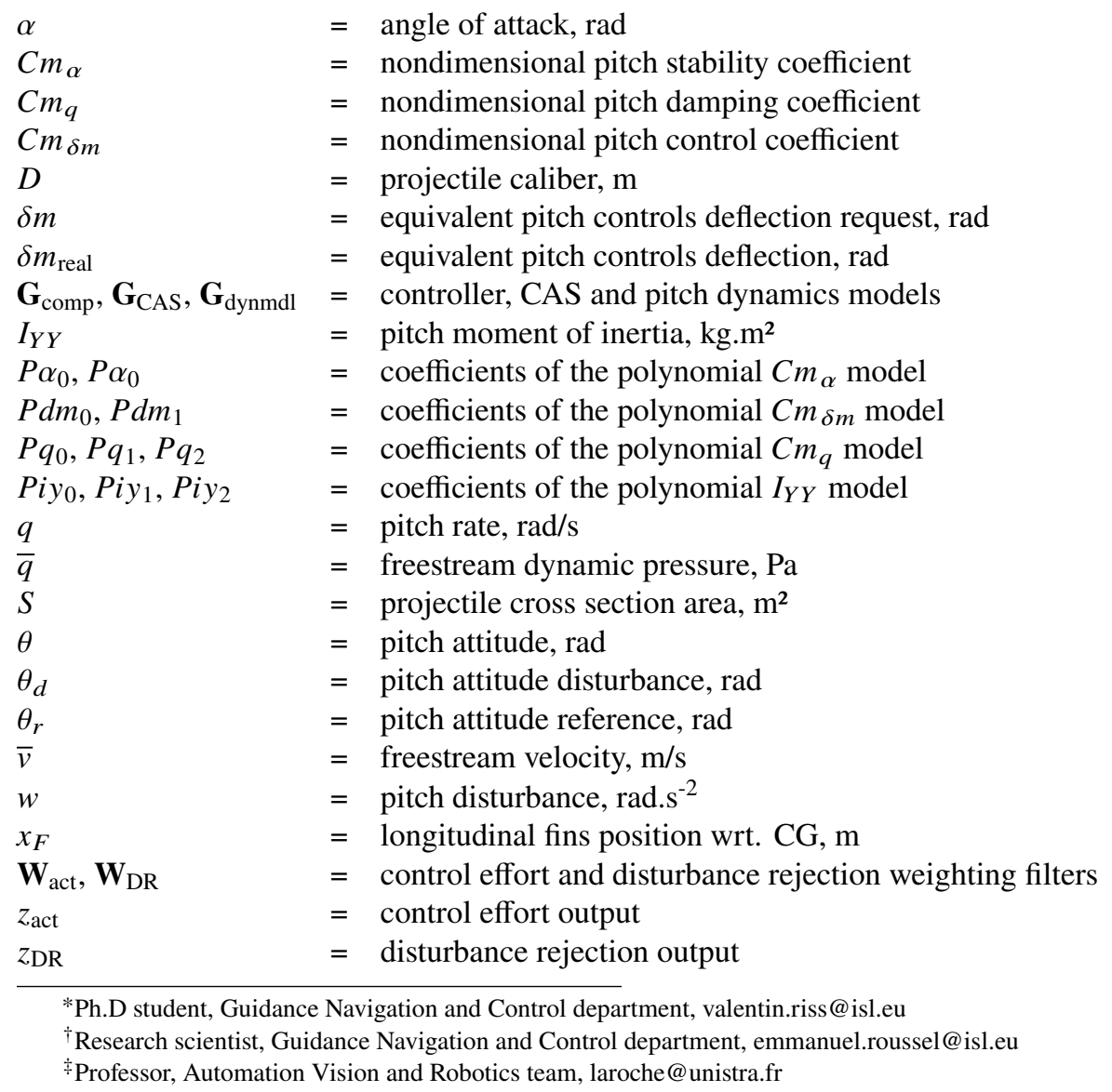




\section{Introduction}

\section{A. Context: towards long-range agile projectiles}

In the last decades, guided projectiles have been developed as a means to improve accuracy and lethality of artillery fires [1]. Subsequent efforts have also aimed at stretching the sphere of action of land- and surface-based systems by extending the range of guided ammunition [2]. Innovative projectile designs [3] [4] have been developed in order to achieve increased range through a glide phase performed at a shallow flight path angle.

In urban warfare or non-line-of-sight firing scenarios, a top attack is required in order to to clear obstacles and terrain features such as elevated buildings or treelines. Moreover, it is also beneficial to warhead lethality as some targets like armored vehicles are more vulnerable when struck from above. Thus, a scenario combining a shallow glide phase and a top attack, as pictured in fig. 1, is of particular interest.

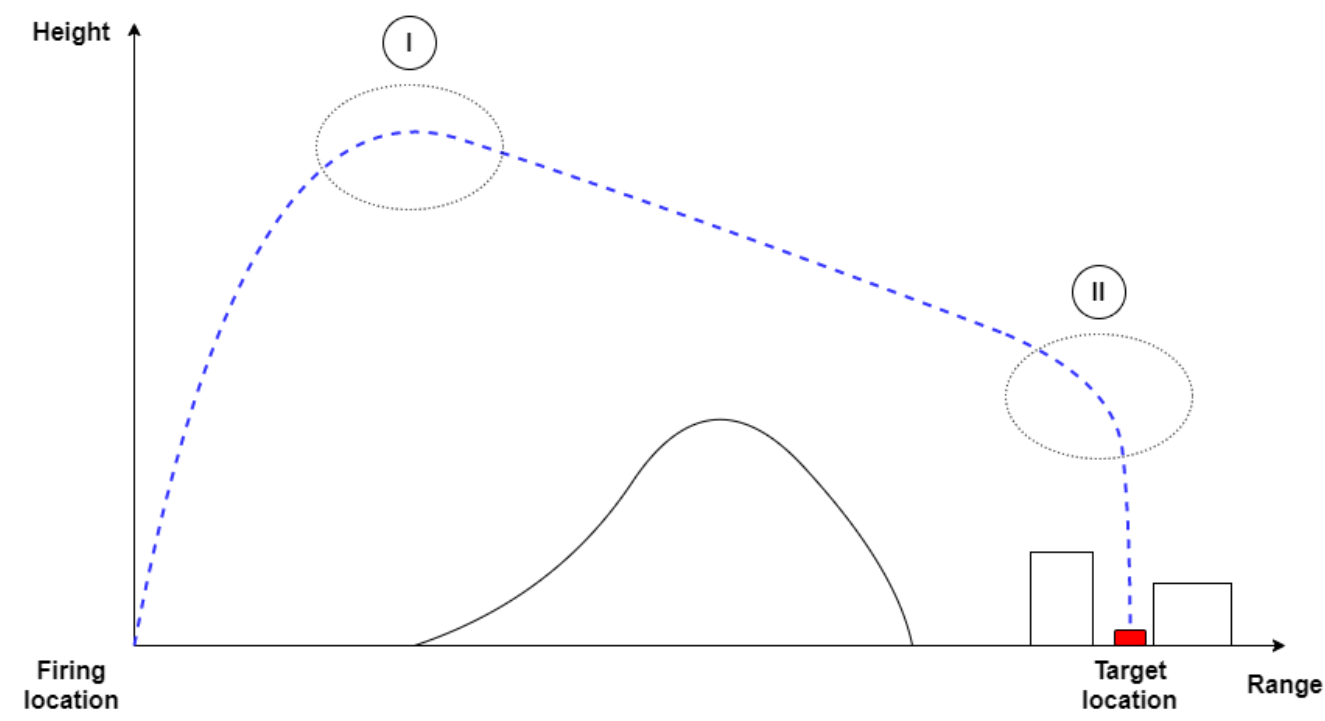

Fig. 1 Combined gliding flight and top attack scenario

Two maneuvers are required in order to follow the above-mentioned flight profile. First, at the apogee (see fig. 1 label I), the projectile should transition from ballistic to gliding flight. Due the low dynamic pressure stemming from the combination of high altitude and low airspeed, the projectile may need a substantial lift coefficient to follow the desired trajectory. At the beginning of the terminal phase, it is necessary to swiftly transition from a glide to a steep dive by the means of an aggressive pitching maneuver (see fig. 1 label II). A considerable load factor is required in order to minimize the turn radius and avoid obstacles.

Both gliding projectile designs mentioned in the first paragraph feature large lifting surfaces in order to maximize their lift-to-drag ratios. Compared to classical guided projectiles such as artillery shells fitted with course correction fuses, these additional surfaces enable the gliding projectiles to generate significant lift by increasing their angle of attack (AoA) [3] instead of relying solely on the forces produced by their control surfaces.

As a result, precise pitch attitude control is crucial to ensure maximum maneuvering performance and accurate flight path tracking in the above-mentioned scenario. In order to do that, the available control moment should be sufficient to generate adequate pitch rate and angle of attack. This poses a challenge as available control force and actuator bandwidth are usually very limited for this type of airframe [5] due to the design constraints on the control and actuation system (CAS) such as G-hardening, packaging space, power usage and unit cost. Thus, actuator rate and deflection limits are susceptible to be reached in normal operation. This must be avoided as, without proper anti-windup schemes, it may degrade controller performance and even lead to closed-loop instability [6]. 


\section{B. Plant-Controller Optimization}

In order to mitigate the actuator limitations, the tradeoff between pitch authority (which conditions the amplitude of control surfaces deflections) and stabilization effort (that drives the requirements on actuator bandwidth) is of primary interest. This compromise is driven by the static stability coefficient of the projectile: The $C m_{\alpha}$ not only quantifies the magnitude and direction of the pitching moment generated in response to an angle of attack disturbance, it also conditions the size of the trim map. This is because, for a given control order $\delta m$, the equilibrium pitch attitude depends on the ratio of $C m_{\alpha}$ and $C m_{\delta m}$.

Legacy methods for determining the adequate amplitude of the static stability coefficient are based on open-loop stability criteria and thus may be excessively conservative in the frame of closed-loop control [7]. More recently, Fresconi et al. [8] conducted a parametric study of the influence of various actuation schemes, control laws and geometric parameters on projectile range. Results showed that center of gravity $(\mathrm{CG})$ position, which determines static stability, had a significant influence on performance. However, to the best of the authors' knowledge, this relationship has not been implemented in a projectile design framework.

In this paper, the question of tuning the static stability of the projectile in order to improve its maneuverability is addressed through a plant-controller optimization problem. This approach, also known as codesign, consists in tuning both controller gains and physical parameters influencing the open-loop plant dynamics. It has been applied to a variety of domains such as chemistry [9], robotics [10] and powertrains [11][12]. In aerospace, the method has mainly been used in preliminary design for different purposes such as mass reduction of large flexible structures [13], reduction of avionics requirements for satellite attitude control [14], optimization of an airborne-wind-energy system [15] and aircraft control surfaces sizing under handling qualities constraints [16] [17].

In the latter case, Niewhoener and Kaminer [16] expressed the requirements as a constrained optimization problem which was solved using the linear matrix inequality (LMI) framework. Alazard et al. [14] introduced simultaneous optimization of both plant and controller parameters in a single, fixed-structure $\mathcal{H}_{\infty}$ synthesis problem. Denieul et al. [17] applied this methodology to elevons sizing and control allocation for a blended-wing-body aircraft concept.

The benefit of the integrated design of aerodynamics and control over the sequential "design then control" process is twofold. First, as shown in the pioneering work of Fathy [18], solving the design and control problem successively does not guarantee optimal system performance. This could only be achieved by using nested or simultaneous optimization strategies. In our case, it means that adjusting the lifting surfaces sizes assuming a fixed set of control gains and then tuning the gains to perform best with the tailored airframe will likely lead to worse closed-loop performance than simultaneously tuning both sets of parameters.

Also, concurrent design of airframe geometry and controller structure allows to mitigate risks and avoid unexpected redesigns of the aerodynamic configuration. This could be the case if the airframe proves to be excessively stable and requires too much control force to trim or, alternatively, if its lack of open-loop stability results in excessive actuator bandwidth requirements.

This work proposes an original application of the plant-controller optimization process to tailor the static stability of a fin-stabilized guided projectile in order to minimize actuator usage under control performance requirements. Section III describes the modeling process used to capture the projectile pitch dynamics. In section IV the setup and resolution of the optimization problem are presented. In light of the subsequent results, the relevance of the plant-controller optimization scheme is assessed in section $\mathrm{V}$.

\section{Experimentally-infused projectile modeling}

This section introduces the geometry of a simplified long-range fin-stabilized projectile. This aerodynamic configuration is described by a set of geometric parameters which are partially included into the PCO framework. Then, the experimental setup used for model identification is presented and a linear parametric model of the projectile pitch dynamics is proposed.

\section{A. Parametric projectile geometry}

The projectile geometry pictured in fig. 2 features a cylindrical body with an hemispherical nose as well as two sets of lifting surfaces named fins and canards. The canards are small actuated surfaces placed forwards of the center of gravity, designed to generate pitching moment in order to control the projectile attitude. The fins are larger surfaces 
located aft of the CG which provide static stability and generate most of the lift force. The dimensions of both sets of surfaces are detailed in table 1 along with some important geometrical characteristics of the mockup.

The longitudinal position of the finned sleeve has been chosen as the geometrical parameter to be optimized in conjunction with the controller gains. Fins represent approximately $70 \%$ of the lifting surfaces area and are located further away of the CG than the canards, thus their position has a significant influence on the location of the projectiles center of pressure and its static stability. Moreover, it is desirable to find a geometric parameter that has little influence on the aerodynamic performance of the projectile in order not to interfere with other design criteria which are outside the scope of this study, such as maximum lift-to-drag ratio. In that regard, varying the fins position do not affect their area nor their aspect ratio so the projectile aerodynamic performance should not be significantly affected.

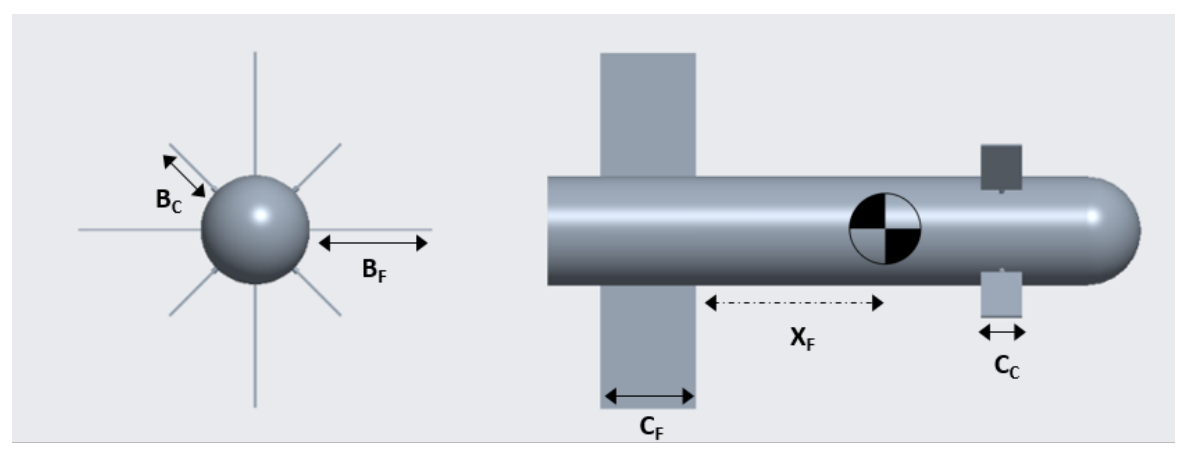

Fig. 2 Parametric projectile geometry (dashed arrow shows adjustable fins position)

\begin{tabular}{|c|c|c|}
\hline Mass: $2560 \mathrm{~g}$ & Number of fins: 4 & Number of canards: 4 \\
\hline Tot. length: $435 \mathrm{~mm}$ & Fins chord $\mathbf{C}_{\mathbf{F}}: 45 \mathrm{~mm}$ & Canards chord $\mathbf{C}_{\mathbf{C}}: 30 \mathrm{~mm}$ \\
\hline Caliber: $80 \mathrm{~mm}$ & Fins span $\mathbf{B}_{\mathbf{F}}: 90 \mathrm{~mm}$ & Canards span $\mathbf{B}_{\mathbf{C}}: 60 \mathrm{~mm}$ \\
\hline CG pos. from nose: $187 \mathrm{~mm}$ & Fins pos. $\mathbf{X}_{\mathbf{F}}$ wrt. CG* $: 122$ to $200 \mathrm{~mm}$ & Canards pos. wrt. CG*: $-99 \mathrm{~mm}$ \\
\hline
\end{tabular}

* measured from the surface leading edge

Table 1 Projectile mockup geometry
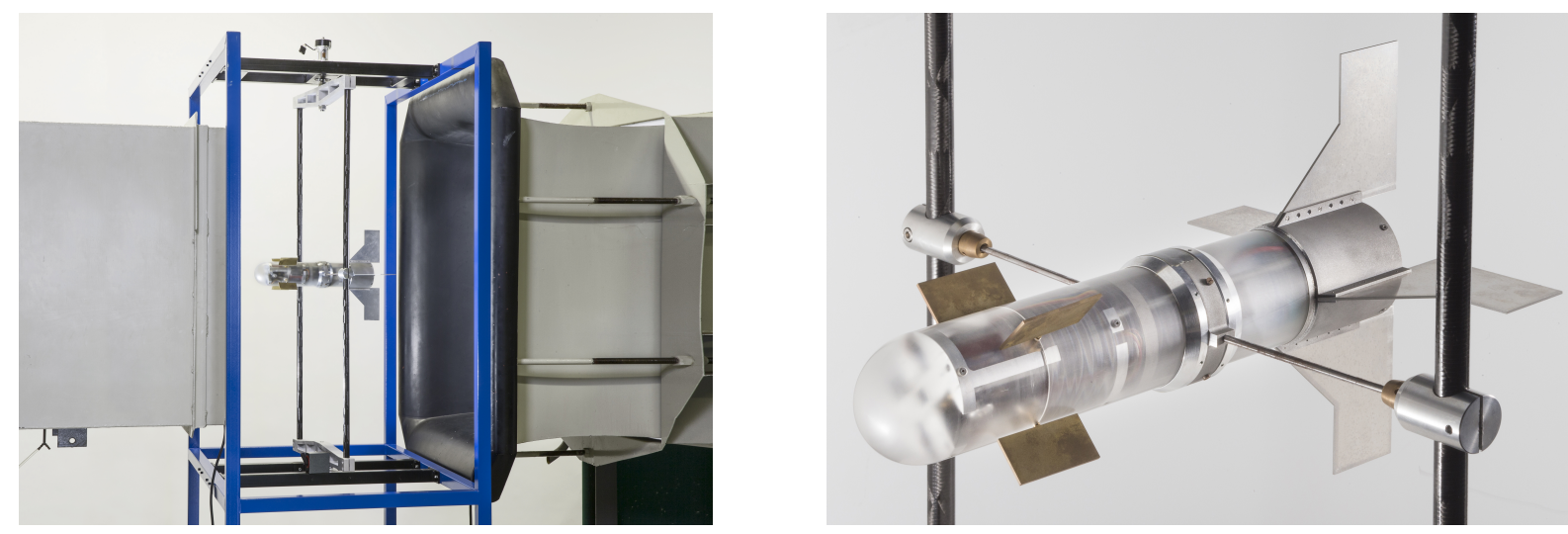

Fig. 3 The ACHILES experimental setup (reproduced from [19] with the author's permission) 


\section{B. Experimental setup}

The ACHILES (Automatic Control Hardware-In-the-Loop Experimental Setup) is a modeling, identification and control framework previously developed at the French-German research institute of Saint-Louis. It allows open-loop angular dynamics identification and closed-loop testing of attitude control laws. Both types of experiments are performed using an instrumented and actuated projectile mockup mounted on a 3 degrees-of-freedom gimbal (see fig. 3). The projectile and its support structure are placed in the test section of a closed return subsonic wind tunnel, where the attitude dynamics are excited by deflecting the canards. Euler angles and angular rates are measured by an onboard inertial measurement unit augmented with a yaw encoder while remote control and real-time monitoring is performed using Simulink. A comprehensive description of the hardware and software implementations of the ACHILES is available in Strub's previous work [20] [19].

\section{Estimation of the aerodynamic coefficients}

A data-based approach has been retained, leveraging on the ACHILES modeling framework to provide accurate estimates of the projectile aerodynamic coefficients. For each fins position, a linear model of the projectile pitch dynamics was identified from the linearized equations of rotational motion. As the projectile mockup is gimbaled inside the wind tunnel, its flight path angle is always zero and its pitch attitude $(\theta)$ can be substituted for its angle of attack $(\alpha)$. Thus, under additional assumptions detailed in the author's previous work [21], the linearized equation of pitching motion is given by:

$$
I_{Y Y} \ddot{\theta}=\bar{q} S D\left(C m_{\alpha} \theta+C m_{q} \frac{q D}{\bar{v}}+C m_{\delta m} \delta m\right)
$$

Data collection experiments were carried out using a similar process as described in [21]. The open-loop projectile was subjected to a series of steps of constant amplitudes but variable lengths in order to excite the projectile dynamics over the frequency range of interest. The equivalent pitch controls deflection was used as an input while the pitch attitude and pitch rate outputs were measured. One minor difference consisted in the reduction of the mockup degrees of freedom by locking the roll axis and preventing any excitation in yaw. Due to the gimbal frame moment of inertia (MOI), the bandwidth of the yaw axis is significantly lower than on the other axes. Thus, the yaw axis could be left free in order to compensate for a minor misalignment in the support structure. Each experiment was performed at a different fins position in order to sweep the airframe design space. When adjusting the fins, the CG position had to be kept constant by the means of a counterweight to avoid disturbing the static balance of the mockup in its gimbal. Due to this balancing constraint, the interval of fins positions that could be tested was restricted to [122 $\mathrm{mm}, 200 \mathrm{~mm}]$ with regard to the CG.

Finally, the input, attitude and rate measurements were fed into a prediction error minimization algorithm [22] in order to estimate the projectile aerodynamic coefficients $\mathrm{Cm}_{\alpha}, \mathrm{Cm}_{q}$ and $\mathrm{Cm}_{\delta m}$ for each position of the fins. The grey-box model structure was based on equation 1 and assumed prior knowledge of the projectile moment of inertia which had previously been measured with laboratory equipment.

\section{Linear parametric model of pitch dynamics}

In order to perform airframe-controller optimization, the open-loop pitch dynamics of the projectile must be described for any value of the geometric parameter (the longitudinal fins position) within its variation range. The set of coefficients gathered with the above-mentioned process was used to derive metamodels of the aerodynamic coefficients as polynomial functions of the fins position. The order of the polynomial metamodels were chosen to faithfully capture the trend of the parametric variation of each coefficient while retaining a low order to minimize the impact of measurement and estimation errors. Figures 4 , 5 and 6 show the fits of the $\mathrm{Cm}_{\alpha}, \mathrm{Cm}_{q}$ and $\mathrm{Cm}_{\delta m}$ metamodels on their respective set of identified coefficients. 


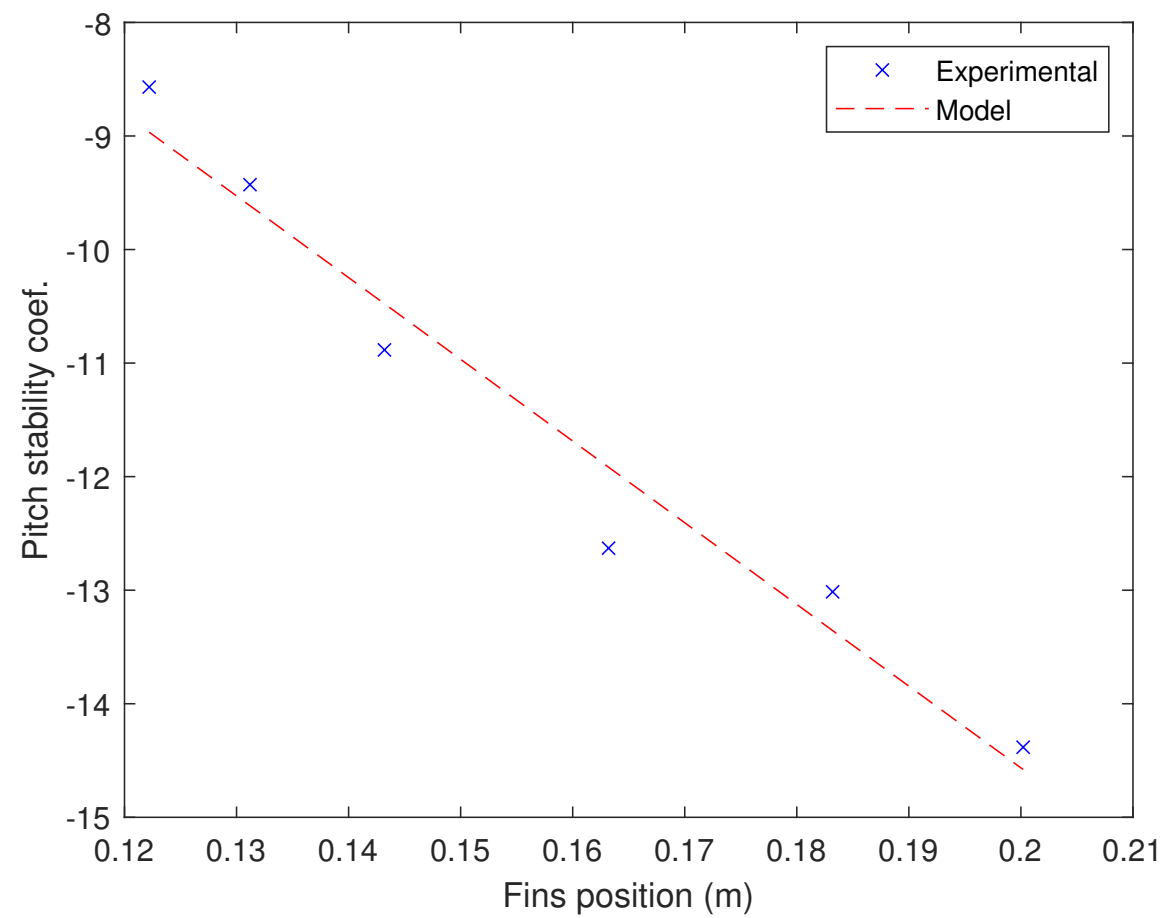

Fig. $4 \mathrm{Cm}_{\alpha}$ metamodel fit on identification data

The parametric models of the aerodynamic coefficients were complemented by a polynomial model of the pitch moment of inertia which was constructed using the parallel axis theorem along with mass and MOI measurements. All of these models are given in the set of equations below:

$$
\left\{\begin{array}{l}
C m_{\alpha}\left(x_{\mathrm{F}}\right)=P \alpha_{0}+P \alpha_{1} x_{\mathrm{F}} \\
C m_{\delta m}\left(x_{\mathrm{F}}\right)=P d m_{0}+P d m_{1} x_{\mathrm{F}} \\
C m_{q}\left(x_{\mathrm{F}}\right)=P q_{0}+P q_{1} x_{\mathrm{F}}+P q_{2} x_{\mathrm{F}}^{2} \\
I_{\mathrm{YY}}\left(x_{\mathrm{F}}\right)=P i y_{0}+P i y_{1} x_{\mathrm{F}}+P i y_{2} x_{\mathrm{F}}^{2}
\end{array}\right.
$$

Both aerodynamics and inertia metamodels are combined into a state-space structure to form the linear, parameterdependent model of the projectile pitch dynamics. The model has two states $(\theta$ and $q)$, two inputs $(\delta m$ and $w)$ and a single output $(\theta)$. The state-space representation of the projectile model is given by the following equation:

$$
\left[\begin{array}{c}
\dot{\theta} \\
\dot{q}
\end{array}\right]=\left[\begin{array}{cc}
0 & 1 \\
A_{m \theta}\left(x_{\mathrm{F}}\right) & A_{m q}\left(x_{\mathrm{F}}\right)
\end{array}\right]\left[\begin{array}{l}
\theta \\
q
\end{array}\right]+\left[\begin{array}{cc}
0 & 0 \\
B_{m \delta m}\left(x_{\mathrm{F}}\right) & 1
\end{array}\right]\left[\begin{array}{c}
\delta m \\
w
\end{array}\right]
$$

with the coefficients:

$$
A_{m \theta}\left(x_{\mathrm{F}}\right)=\bar{q} S D \frac{C m_{\alpha}\left(x_{\mathrm{F}}\right)}{I_{Y Y}\left(x_{\mathrm{F}}\right)} \quad A_{m q}\left(x_{\mathrm{F}}\right)=\bar{q} S D^{2} \frac{C m_{q}\left(x_{\mathrm{F}}\right)}{\bar{v} I_{Y Y}\left(x_{\mathrm{F}}\right)} \quad B_{m \delta m}\left(x_{\mathrm{F}}\right)=\bar{q} S D \frac{C m_{\delta m}\left(x_{\mathrm{F}}\right)}{I_{Y Y}\left(x_{\mathrm{F}}\right)}
$$




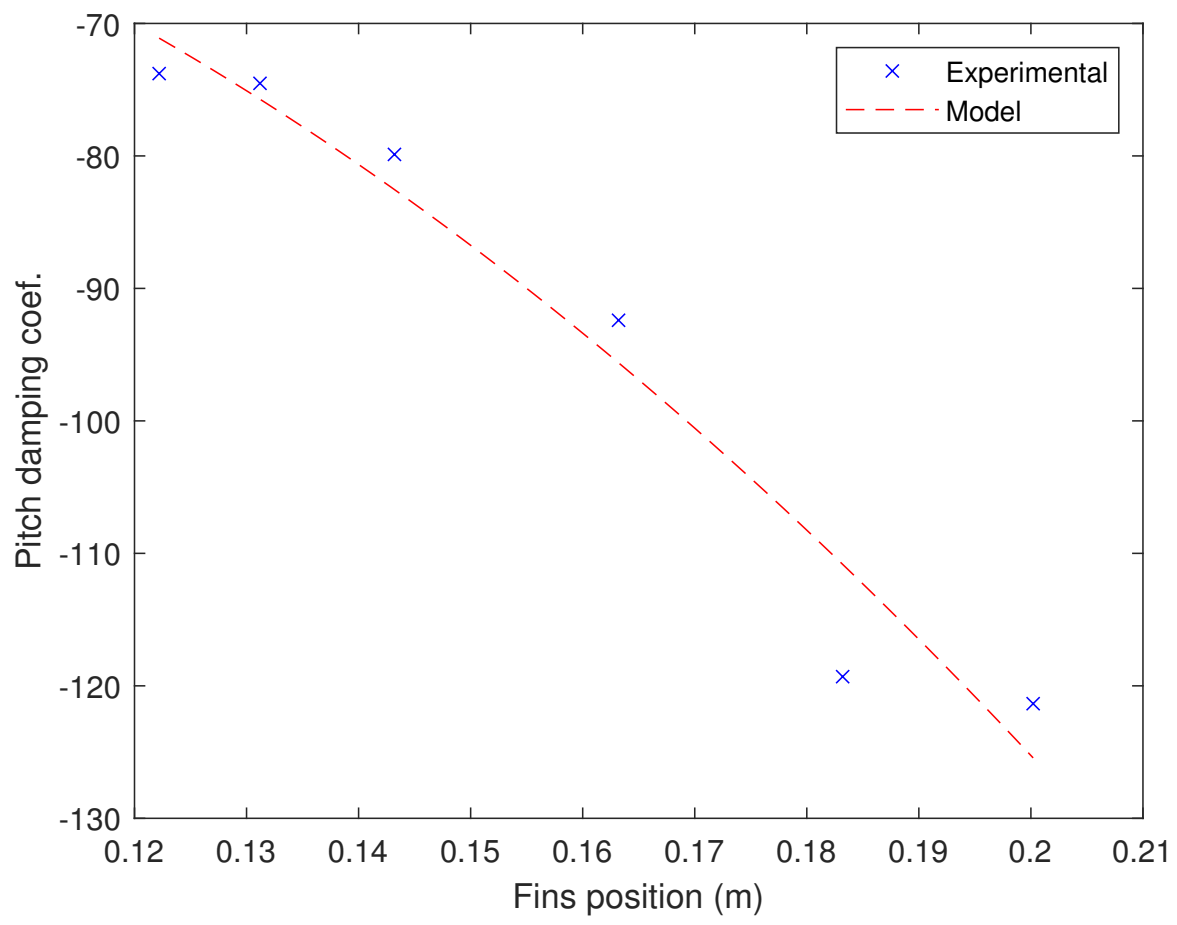

Fig. $5 \mathrm{Cm}_{q}$ metamodel fit on identification data

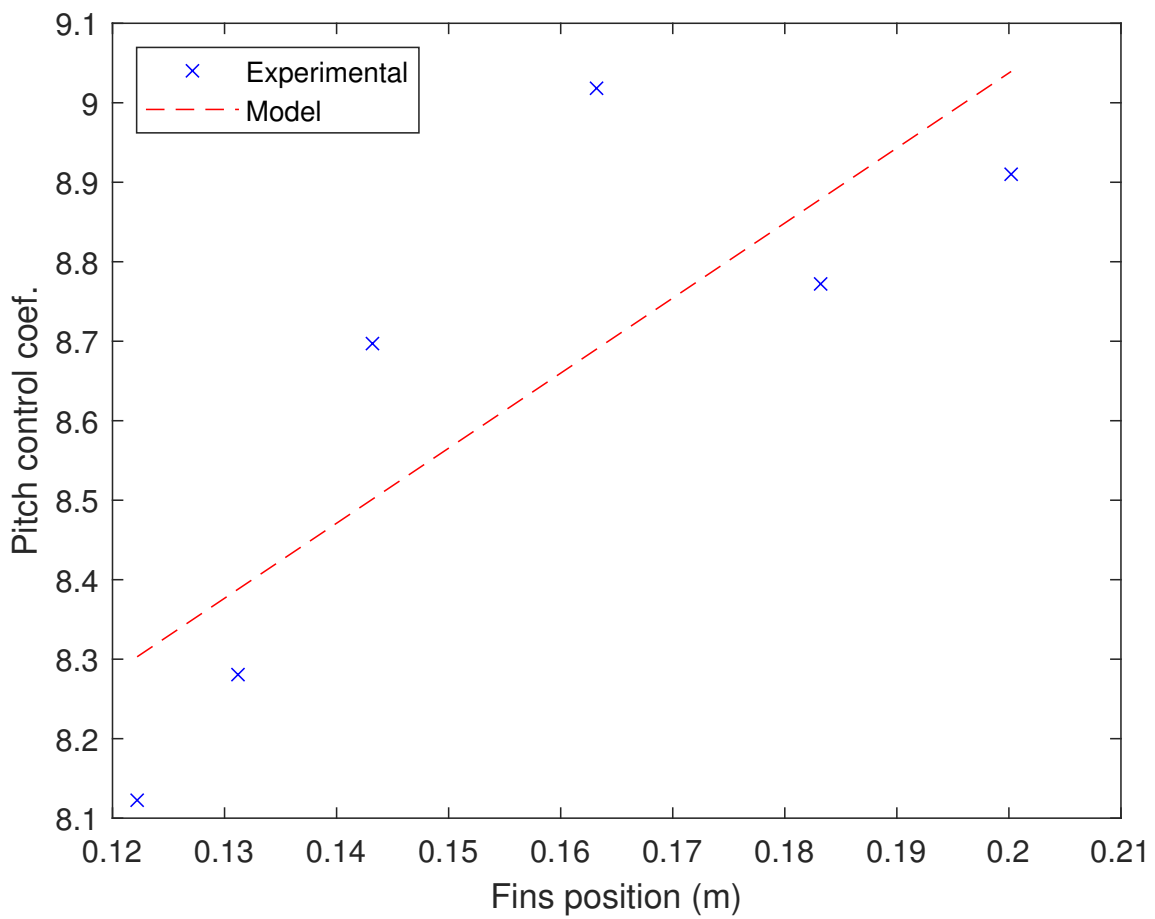

Fig. $6 C m_{\delta m}$ metamodel fit on identification data 


\section{Plant-Controller Optimization problem}

This section details the setup and implementation of the plant-controller optimization problem. The aim is to assess the interest of plant-controller optimization in the frame of a simplified pitch attitude control problem, where the set of geometric parameters to be adjusted has been restricted to the fins longitudinal position. The cost function and optimization constraints are chosen and implemented in the multi-objective structured $\mathcal{H}_{\infty}$ synthesis framework pictured in figure 7

Due to the inclusion of the geometric parameter into the controller synthesis framework, the resulting problem is nonsmooth [16]. The MATLAB Systune routine is used to solve the optimization problem as it is able to handle such nonsmooth and nonconvex scenarios. However, convergence to a global minimum cannot be guaranteed and multiple initializations should be performed in order to maximize the chance of reaching the global optimum. The details of the solving algorithms and their implementation are given in references [23] and [24].

The plant model includes the parametric pitch dynamics model described in the previous section, augmented with a third-order model of the CAS dynamics found in [19]. The controller is implemented in state-space form with a single input $\left(\theta_{e}\right)$ and 8 states. The controller order is chosen to be equal to the order of the augmented plant (plant model including CAS dynamics and frequency templates used for $\mathcal{H}_{\infty}$ synthesis). As a perspective, the controller order could be reduced in order to facilitate its implementation.

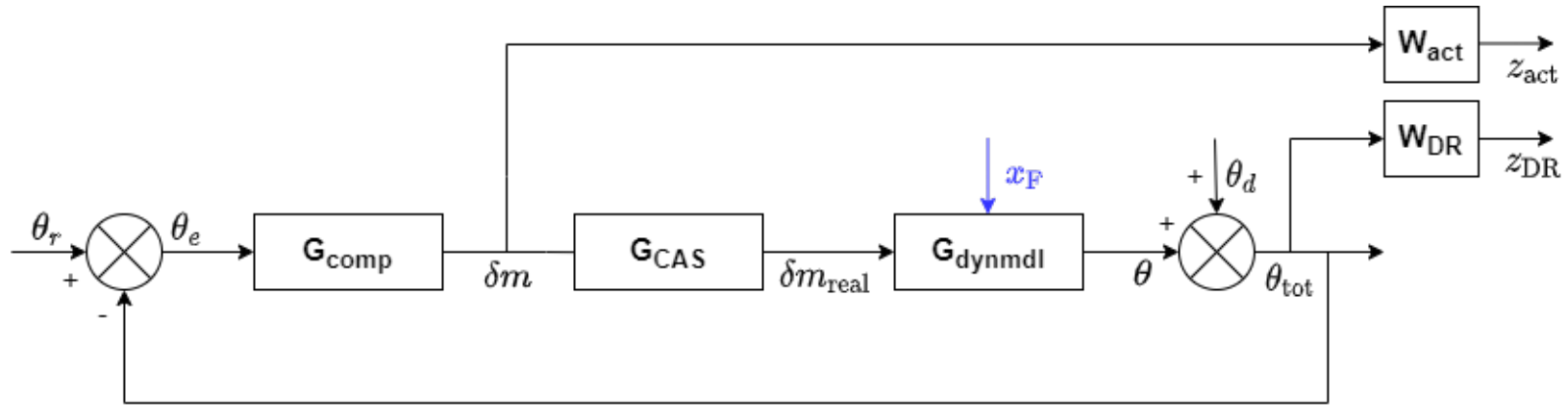

Fig. 7 Multi-objective $\mathcal{H}_{\infty}$ synthesis framework

The cost function and optimization constraints are based on frequency templates commonly used in the $\mathcal{H}_{\infty}$ synthesis framework. The objective of the plant-controller design is to minimize the control effort while ensuring adequate controller performance. Actuator usage is monitored by the closed-loop transfer $T_{\theta r \rightarrow \delta m}$ from the pitch attitude reference to the actuator input, while performance is represented by a disturbance rejection constraint using the output sensitivity function $T_{\theta d \rightarrow \theta \text { tot }}$. These transfers are multiplied by their respective weighting filters $W_{\text {act }}$ and $W_{\mathrm{DR}}$ to form the actuator usage $z_{\text {act }}$ and disturbance rejection $z_{\mathrm{DR}}$ channels. As $T_{\theta d \rightarrow \theta \text { tot }}=T_{\theta r \rightarrow \theta e}$, this setup is equivalent to a classical S/KS synthesis framework.

The weighting filters $W_{\text {act }}$ and $W_{\mathrm{DR}}$ are chosen as first order lead-lag filters characterized by their respective low-frequency gain, high-frequency gain and $-3 \mathrm{~dB}$ bandwidth. The frequency template $1 / W_{\mathrm{DR}}$ pictured in fig. 8 is shaped like a high-pass filter in order to provide good reference tracking and disturbance rejection performance. $1 / W_{\text {act }}$ is shown on fig. 9 and features low-pass behavior to attenuate the effect of measurement noises, preserve the actuators from high frequency content and limit the control energy. The low-frequency bound on $W_{\mathrm{DR}}$ is set to a very small nonzero value in order to suppress steady-state error. The filter bandwidth is set to $3 \mathrm{rad} / \mathrm{s}$ which represents an achievable objective for this system as demonstrated in previous work [21]. The high-frequency asymptote is a compromise between performance and actuator usage, thus it is set to a conservative value of 1.3 to avoid overconstraining the control effort minimization problem. As for $W_{\text {act }}$, the magnitude of the low frequency gain is bounded by $2(+6 \mathrm{~dB})$ to avoid excessive canard deflection amplitudes. The bandwitdh limit is set to $10 \mathrm{rad} / \mathrm{s}$ while the high-frequency asymptote has a small non-zero value to cut off the high frequency content of the control signal.

The optimization constraints are computed by taking the $\mathcal{H}_{\infty}$ norm of $T_{\theta r \rightarrow z \text { act }}$ and $T_{\theta d \rightarrow z_{\mathrm{DR}}}$. The actuator usage constraint is declared as a soft constraint, which means that the algorithm will attempt to minimize $\left\|T_{\theta r \rightarrow z a c t}\right\|_{\infty}$ beyond one. Conversely, the performance constraint is implemented as a hard constraint so that the optimization routine will stop once $\left\|T_{\theta d \rightarrow z_{\mathrm{DR}}}\right\|_{\infty}$ is smaller than one. In that case, it is guaranteed that $T_{\theta d \rightarrow \theta \text { tot }}$ fits inside its prescribed template 
for all frequencies. The plant-controller design that minimizes the soft constraint while fulfilling the hard constraint is retained and $\left\|T_{\theta r \rightarrow z \text { act }}\right\|_{\infty}$ is used as a cost function in order to compare the quality of different solutions (cf. table 2).

\section{Optimization results}

In this section, the results of the control effort minimization problem are presented and compliance with performance constraints is checked. Then, the results of the codesign approach are compared with the outcomes of the traditional "design then control" methodology in order to assess the relevance of the airframe-controller optimization scheme.

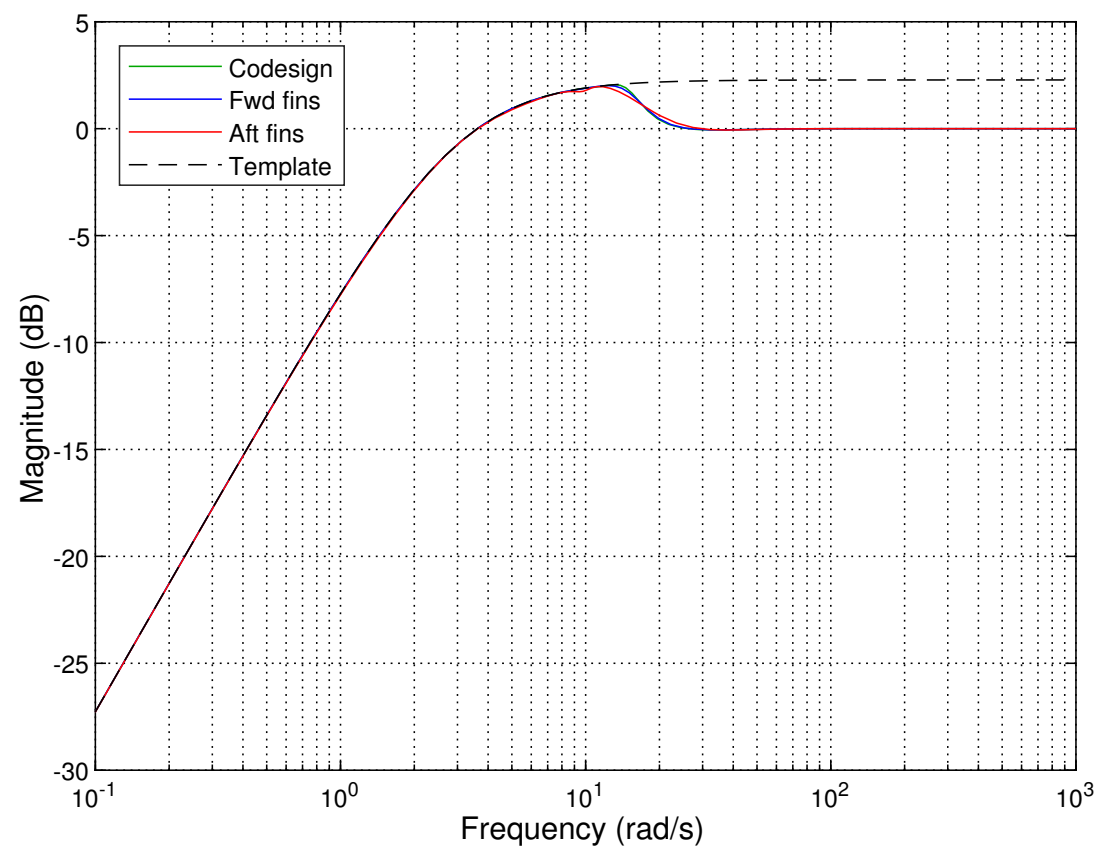

Fig. 8 Disturbance rejection transfer $T_{\theta d \rightarrow \theta \text { tot }}$ and template $1 / W_{\mathrm{DR}}$

Figures 8 and 9 respectively show $T_{\theta d \rightarrow \theta \text { tot }}$ and $T_{\theta r \rightarrow \delta m}$ for three different designs. In the first case, the geometric parameter is left free and simultaneous plant-controller optimization is performed. In the other two cases, the fins are fixed to their maximum forward (122 $\mathrm{mm}$ behind CG) and aft (200 $\mathrm{mm}$ behind CG) positions while the controller gains are tuned. The sensitivity plot of fig. 8 demonstrates that the optimizer is able to find airframe-controller designs that fulfill the hard constraint on disturbance rejection performance for each of the three fins positions. Figure 9 shows two critical areas where the frequency responses lie close to the template, which are the static gain and the high frequency roll-off. In the latter case, the optimal configuration is superior to both forward and aft fins designs as its high frequency gain asymptote is offset downwards.

Three figures of merits presented in table 2 are retained to quantify the actuator usage of a given airframe-controller design. The first one is the cost function $\left\|T_{\theta r \rightarrow z a c t}\right\|_{\infty}$ derived from the reference to actuator transfer $T_{\theta r \rightarrow \delta m}$. Given the remarks on fig. 9, the static gain and $-25 \mathrm{~dB}$ crossover frequency of this transfer have been retained as additional metrics to compare the three configurations in the frequency ranges where the actuator limitations are the most stringent. The crossover frequencies confirm that the optimal design presents the best roll-off characteristics. However, the table also shows that this design has a higher static gain than the forward fins configuration. This is a drawback as it implies that larger fins deflections are required to achieve a given trim attitude.

Figure 10 depicts the variation of the cost function $\left\|T_{\theta r \rightarrow z a c t}\right\|_{\infty}$ as the parametric design space is swept. Each data point corresponds to a design where the controller has been tuned for a specific airframe with a given fins position. The optimal fins position is found $149 \mathrm{~mm}$ behind the center of mass, close to the middle of the parametric range allowed by the model. Thus, the best configuration is neither the most stable airframe nor the least stable one and the solution of the control effort minimization problem is non-trivial. As confirmed by table 2 , the optimal airframe-controller 


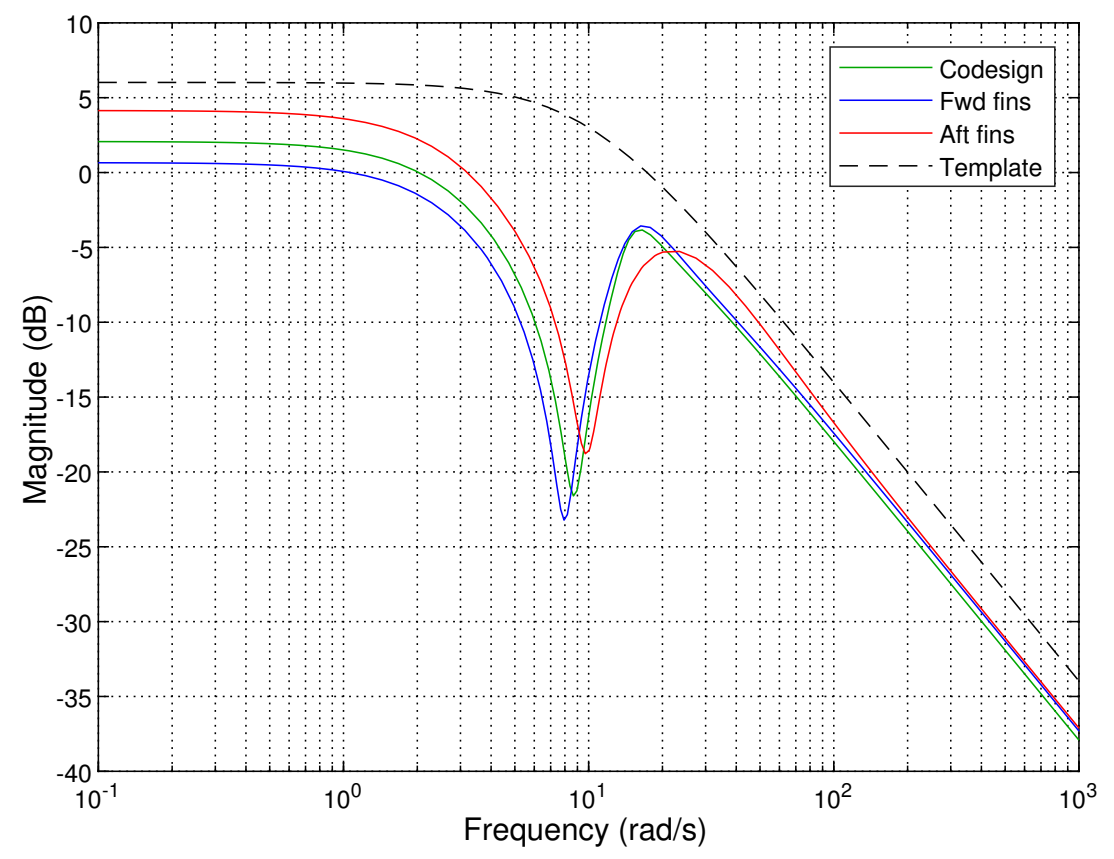

Fig. 9 Control effort transfer $T_{\theta r \rightarrow \delta m}$ and template $1 / W_{\text {act }}$

\begin{tabular}{|c|c|c|c|}
\hline Criterion & Opt. fins & Fwd. fins & Aft fins \\
\hline$\left\|T_{\theta r \rightarrow z \text { act }}\right\|_{\infty}$ cost function & 0.6347 & 0.6809 & 0.8056 \\
\hline$T_{\theta r \rightarrow \delta m}$ static gain & 1.269 & 1.079 & 1.611 \\
\hline$T_{\theta r \rightarrow \delta m}-25$ db crossover freq. $(\mathrm{Hz})$ & 35.89 & 38.47 & 39.74 \\
\hline
\end{tabular}

Table 2 Actuator usage metrics

design allows noticeable improvement of the cost function over both extreme fins configurations. The scatter at the beginning of the fins position interval confirms the nonconvex and nonsmooth nature of the optimization problem. It is interesting to note that the solution found by the codesign routine may be slightly worse than the solution of the control problem for a neighboring fins position. In fact, as the geometric parameter is fixed and the controller is full order, the $\mathcal{H}_{\infty}$ synthesis problem is convex. This is no longer the case when the geometric parameter is left free, that is why the solution of the codesign problem may be more conservative. However, in this case, the PCO routine gives better results than the manual sweep of the airframe design space. It is also much less computationally intensive, as it only solves the synthesis problem once instead of looking for the optimal gains associated with each specific airframe. On a high-performance laptop, solving the codesign problem takes in average 12 seconds while sweeping the parameter space with 50 synthesis points like in fig. 10 requires approximately 8 minutes and 8 seconds, which is more than 40 times longer.

To sum up, this case study reveals that the optimal airframe-controller design allows to reduce the actuator usage compared to arbitrary aerodynamic configurations. Moreover, the ACO methodology has been found to be significantly less computationally intensive than solving the control problem for a large set of fins positions. Consequently, the codesign approach is relevant in the frame of long-range guided projectile design and control. 


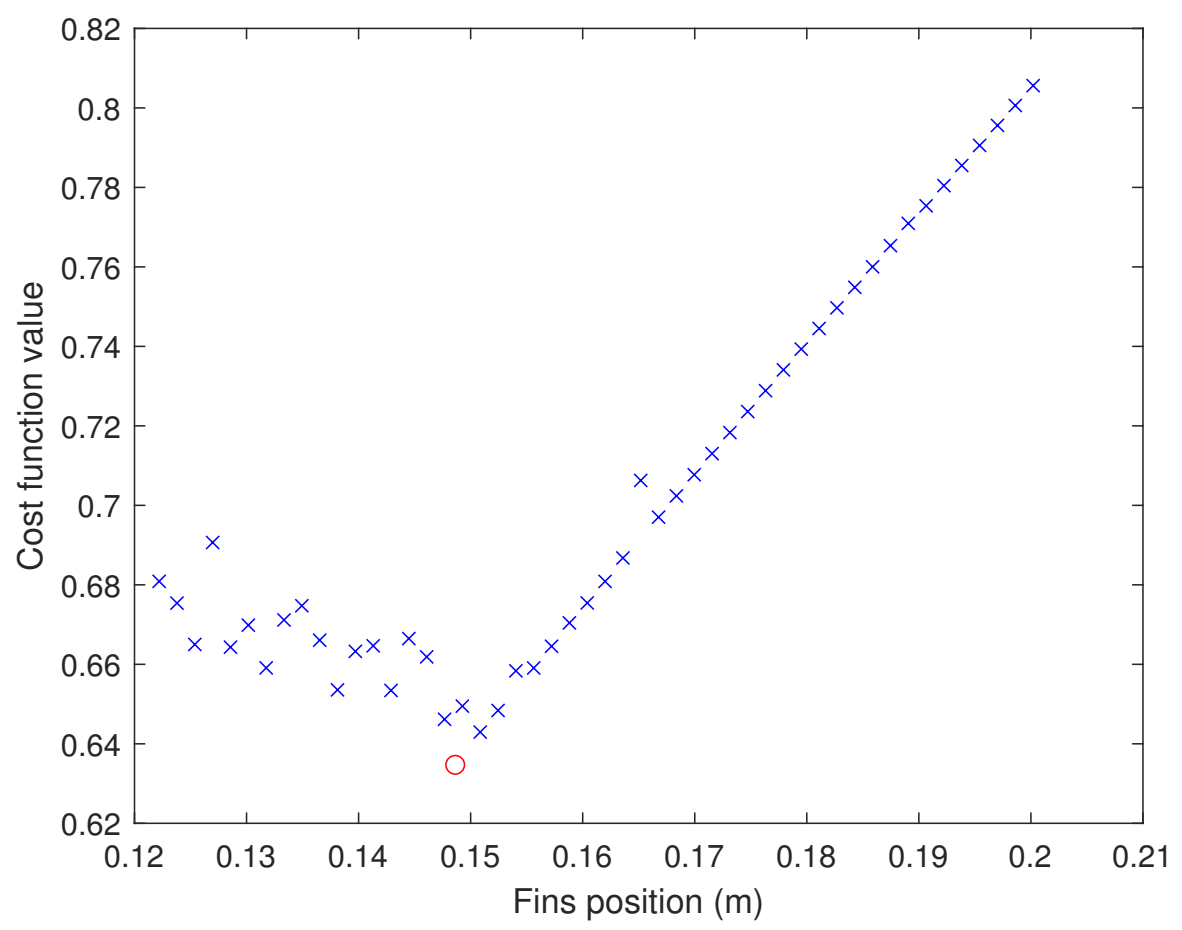

Fig. 10 Cost function for different airframe-controller designs (the optimal design is represented by a red circle)

\section{Conclusion}

\section{A. Summary}

This paper investigates the use of plant-controller optimization in the frame of guided projectile control. The interest of tailoring the static stability of a long-range finned projectile is shown. A linear parametric model of the pitch dynamics is created from experimental data acquired with a bespoke wind-tunnel setup. The projectile fins position is added to the controller synthesis framework as a tunable parameter in order to form a plant-controller optimization problem. Results show the relevance of the codesign approach as the optimal airfame-controller design demonstrates reduced actuator usage while ensuring adequate disturbance rejection properties.

\section{B. Perspectives}

Given that the interest and suitability of the plant-controller optimization scheme has been proven, several perspectives stand out in order to refine the methodology, expand its scope and validate its results.

It would be desirable to further decrease actuator bandwidth requirements in order to pave the way for more affordable guided munitions or larger payloads. In that regard, the current optimal design only achieves modest improvement compared to arbitrary geometries. One way of obtaining better quality optima would be to expand the range of fins positions for which the pitch dynamics model is valid. This could be achieved by either conducting more data-collection experiments or revamping the modeling process.

Another perspective for improvement is related to the criteria used to translate the actuator usage requirements. As of now, the actuator deflection is bounded by a static gain limit between the attitude reference and actuator output. In practice, the control authority is limited by the nonlinearity of the canard lift polar at large angles of attack that stems from canard stall. Thus, it would be more accurate to capture the canard stall phenomenon using nonlinear aerodynamics models and derive an adequate constraint or, at least, to validate the optimal design with a nonlinear simulator.

The final outlook aims at improving the robustness and fidelity of the ACO process by introducing closed-loop validation of the optimal design through wind-tunnel tests. The reference tracking and disturbance rejection performance 
of the controlled projectile could be assessed by analyzing its response to a set of input signals (see [21] for an example). The actuators time histories could also be reviewed to quantify actual control effort.

\section{References}

[1] Morrison, P. H., and S. Amberntson, D., "Guidance and control of a cannon-launched guided projectile," Journal of Spacecraft and Rockets, Vol. 14, No. 6, 1977, pp. 328-334.

[2] Costello, M., "Range extension and accuracy improvement of an advanced projectile using canard control," 20th Atmospheric Flight Mechanics Conference, Baltimore, USA, 1995, pp. 324-331.

[3] Decrocq, C., Martinez, B., Albisser, M., Dobre, S., Gnemmi, P., Bailly, Y., and Roy, J.-C., "Aerodynamic prediction of a projectile fitted with fins," International Journal of Numerical Methods for Heat \& Fluid Flow, Vol. 28, No. 5, 2018, pp. $1218-1236$.

[4] Vasile, J. D., Bryson, J., Gruenwald, B. C., Fairfax, L., Strohm, L., and Fresconi, F., "A Multi-Disciplinary Approach to Design Long Range Guided Projectiles," AIAA Scitech 2020 Forum, 2020, p. 1993.

[5] Fresconi, F., "Guidance and control of a projectile with reduced sensor and actuator requirements," Journal of Guidance, Control, and Dynamics, Vol. 34, No. 6, 2011, pp. 1757-1766.

[6] Thai, S., Theodoulis, S., Roos, C., and Biannic, J.-M., "Robust design for the roll-channel autopilot of a canard-guided dual-spin projectile," IFAC-PapersOnLine, Vol. 52, No. 12, 2019, pp. 232-237.

[7] McCoy, R., Modern exterior ballistics: The launch and flight dynamics of symmetric projectiles, Schiffer Pub., 1999.

[8] Fresconi, F., Celmins, I., and Fairfax, L., “Optimal parameters for maneuverability of affordable precision munitions,” 50th AIAA Aerospace Sciences Meeting Including the New Horizons Forum and Aerospace Exposition, 2012, p. 254.

[9] Luyben, M., and Floudas, C., "Analyzing the interaction of design and control-1. A multiobjective framework and application to binary distillation synthesis," Computers \& chemical engineering, Vol. 18, No. 10, 1994, pp. 933-969.

[10] Ravichandran, T., Wang, D., and Heppler, G., "Simultaneous plant-controller design optimization of a two-link planar manipulator," Mechatronics, Vol. 16, No. 3-4, 2006, pp. 233-242.

[11] Reyer, J. A., and Papalambros, P. Y., “Optimal design and control of an electric DC motor," Proceedings of the 1999 ASME Design Engineering Technical Conferences, Citeseer, 1999, pp. 85-96.

[12] Silvas, E., Hofman, T., Murgovski, N., Etman, L. P., and Steinbuch, M., "Review of optimization strategies for system-level design in hybrid electric vehicles," IEEE Transactions on Vehicular Technology, Vol. 66, No. 1, 2016, pp. 57-70.

[13] Hale, A. L., Lisowski, R. J., and Dahl, W. E., "Optimal simultaneous structural and control design of maneuvering flexible spacecraft," Journal of Guidance, Control, and Dynamics, Vol. 8, No. 1, 1985, pp. 86-93.

[14] Alazard, D., Loquen, T., De Plinval, H., and Cumer, C., "Avionics/Control co-design for large flexible space structures," AIAA Guidance, Navigation, and Control (GNC) Conference, 2013, p. 4638.

[15] Nikpoorparizi, P., Deodhar, N., and Vermillion, C., "Modeling, control design, and combined plant/controller optimization for an energy-harvesting tethered wing," IEEE Transactions on Control Systems Technology, Vol. 26, No. 4, 2017 , pp. 1157-1169.

[16] Niewoehner, R., and Kaminer, I., "Integrated aircraft-controller design using linear matrix inequalities," Journal of guidance, control, and dynamics, Vol. 19, No. 2, 1996, pp. 445-452.

[17] Denieul, Y., Bordeneuve, J., Alazard, D., Toussaint, C., and Taquin, G., "Multicontrol Surface Optimization for Blended Wing-Body Under Handling Quality Constraints,” Journal of Aircraft, Vol. 55, No. 2, 2017, pp. 638-651.

[18] Fathy, H. K., Reyer, J. A., Papalambros, P. Y., and Ulsov, A., "On the coupling between the plant and controller optimization problems," Proceedings of the 2001 American Control Conference.(Cat. No. 01CH37148), Vol. 3, IEEE, 2001, pp. 1864-1869.

[19] Strub, G., and Basset, M., "Skid-to-turn autopilot design and validation for an experimental guided projectile prototype," AIAA Guidance, Navigation, and Control Conference, San Diego, USA, 2016, p. 1875.

[20] Strub, G., Gassmann, V., Theodoulis, S., Dobre, S., and Basset, M., "Hardware-in-the-loop experimental setup development for a guided projectile in a wind tunnel," 2014 IEEE/ASME International Conference on Advanced Intelligent Mechatronics, Besançon, France, 2014, pp. 458-463. 
[21] Riss, V., Roussel, E., and Laroche, E., "Attitude Control of a Fin-Stabilized Projectile on a Three-Axis Gimbal in Wind Tunnel," 2020 28th Mediterranean Conference on Control and Automation (MED), IEEE, 2020, pp. 37-43.

[22] Ljung, L., "Prediction error estimation methods," Circuits, Systems and Signal Processing, Vol. 21, No. 1, 2002 , pp. 11-21.

[23] Apkarian, P., and Noll, D., "Nonsmooth $\mathcal{H}_{\infty}$ synthesis," IEEE Transactions on Automatic Control, Vol. 51, No. 1, 2006, pp. 71-86.

[24] Apkarian, P., Ravanbod-Hosseini, L., and Noll, D., “Time domain constrained $\mathcal{H}_{\infty}$-synthesis," International Journal of Robust and Nonlinear Control, Vol. 21, No. 2, 2011, pp. 197-217. 